\title{
A Group Theoretical and Quantum Chemical Study of Electronic Absorption and Fluorescence, Vibrational Spectra, and Conformations of Trimethine Cyanine Dye Molecules
}

\author{
Vita Solomko, ${ }^{1}$ Petro Kondratenko, ${ }^{2}$ and Yuriy Lopatkin ${ }^{1}$ \\ ${ }^{1}$ Department of General and Theoretical Physics, Sumy State University, 2 Rimsky-Korsakov Street, Sumy 40007, Ukraine \\ ${ }^{2}$ Department of Theoretical Physics, Aerospace Institute, National Aviation University, 1 Kosmonavta Komarova Prospekt, \\ Kiev 03680, Ukraine
}

Correspondence should be addressed to Vita Solomko; vita.velikodnaya91@gmail.com

Received 13 October 2015; Revised 30 November 2015; Accepted 8 December 2015

Academic Editor: Sergei Tretiak

Copyright (c) 2016 Vita Solomko et al. This is an open access article distributed under the Creative Commons Attribution License, which permits unrestricted use, distribution, and reproduction in any medium, provided the original work is properly cited.

\begin{abstract}
The energetic structures and conformations of trimethine cyanine dye molecules were investigated. For research, group theoretical and quantum chemical calculation methods were used. The theoretical group analysis of electronic and vibrational structure of molecules was carried out. Also, the energetic structures and conformations of the molecule of this dye were studied. Research shows that the investigated molecule may reside in three different conformational states, one of which is highly symmetric $\left(\right.$ symmetry $\left.C_{2 v}\right)$ and the other two with low symmetry. The third conformer is characterized by lowering of binding energy of the electronic system by $0.23 \mathrm{eV}$, and the long-wavelength absorption band is shifted to lower energies. Also the group theoretical analysis of the trimethine cyanine molecule had allowed systematizing the vibrational and electronic quantum transitions and identifying the bands in the absorption spectra. It is shown that the excitation of the molecule in $S_{1}$-state causes trans-cis-isomerization. The presence of the barrier of $\sim 0.1 \mathrm{eV}$ allows the fluorescence process to compete with isomerization process, but isomerization causes a decrease in the fluorescence quantum yield of the dye.
\end{abstract}

\section{Introduction}

The absorption spectra and fluorescence spectra of solutions of polymethine dyes have been studied quite extensively and described in scientific papers [1-6], since this class of dyes is widely used in practice $[7,8]$. And yet, the energy structure of the molecules of these dyes was not enough studied, which does not allow us to definitely interpret the fluorescence spectra of the higher excited states that are typical of this class of dyes. The conformational structure and way of the transition of the molecule from one conformation to another which affect the quantum yield of fluorescence were not described in detail.

Aims of this work are to describe the results of the investigation of excited states and conformational transformations, for example, model of the molecule, Dioksazoltrimethinecyanine cation (I) (see Scheme 1).

\section{Materials and Methods}

For research, the software package with a powerful graphical user interface for molecular modeling and study of intermolecular interactions HyperChem (Release 8.0.6) was used, in particular the method AM1 [9], which has proved popular in the study of relaxation process of molecules with highly excited states [10-14].

2.1. Theoretical Group Analysis of the Electronic and Vibrational Structure of the Molecule (I). In the ground state molecule (I) has a symmetric structure, which is described by the group of symmetry $C_{2 \gamma}$. For the understanding of the electronic and vibrational structure of this molecule, it was needed to analyze in the framework of group $C_{2 v}$ symmetry of the molecular orbital $(\mathrm{MO})$ and the vibrational modes of the molecule (I). The results of this analysis are shown in Table 1. 
TABLE 1: Table characters of the group $C_{2 v}$ and assignment to him coordinate axes, broadcasts $(T)$, rotation $(R)$, the number of vibrational modes $(n)$, and $\sigma$ - and $\pi$-MO molecule (I).

\begin{tabular}{lccccccccccc}
\hline$C_{2 v}$ & $E$ & $C_{2}$ & $\sigma_{h}$ & $\sigma_{v}$ & & $T$ & $R$ & $N$ & $n$ & $\sigma$ & $\pi$ \\
\hline$A_{1}$ & 1 & 1 & 1 & 1 & $y$ & 1 & 0 & 22 & 21 & 25 & 0 \\
$A_{2}$ & 1 & 1 & -1 & -1 & & 0 & 1 & 10 & 9 & 0 & 6 \\
$B_{1}$ & 1 & -1 & 1 & -1 & $x$ & 1 & 1 & 22 & 20 & 23 & 0 \\
$B_{2}$ & 1 & -1 & -1 & 1 & $z$ & 1 & 1 & 12 & 10 & 0 & 7 \\
$X$ & 1 & -1 & 1 & -1 & & & & & & & \\
$Y$ & 1 & 1 & 1 & 1 & & & & & & & \\
$Z$ & 1 & -1 & -1 & 1 & & & & & & & \\
$T$ & 3 & -1 & 1 & 1 & & & & & & & \\
$R$ & 3 & -1 & -1 & -1 & & & & & & & \\
$N$ & 66 & -2 & 22 & 2 & & & & & & & \\
$\sigma-\mathrm{MO}$ & 48 & 2 & 48 & 2 & & & & & & & \\
$\pi$-MO & 13 & -1 & -13 & 1 & & & & & & & \\
\hline
\end{tabular}<smiles></smiles>

SCHEME 1

It follows from Table 1 that the total number of degrees of freedom $N$ distributed over representations is as follows: $A_{1}$, 22, $A_{2}, 10, B_{1}, 22$, and $B_{2}, 12$. In this work calculations of all vibrational frequencies were carried out. Some of them show themselves in the infrared spectra (polarizations $x, y$, and $z$ ) and Raman spectra $\left(x^{2}, y^{2}, z^{2}, x y, x z\right.$, and $\left.y z\right)$. But inasmuch only fully symmetric vibrations take part in the electronic absorption and fluorescence spectra; these vibrations are given in the table. In Table 2 fully symmetrical vibrations of molecules (I) are shown.

During the formation of the electronic structure of molecules $48 \sigma$-MO and $13 \pi$-MO are formed. In this case, the number of filled electrons MO is 33.8 of which belong to $\pi$-MO, and the rest are in $\sigma-\mathrm{MO}$. All the processes that we shall be interested in occur in the range from MO number 29 to MO number 43. Symmetry and nature of the MO are shown in Table 3.

Considering these data, we can find the polarization of the quantum transitions between separate MO and determine quantum transitions which are forbidden by symmetry (Table 4).

It turned out that quantum transitions with polarization along $z$-axis correspond to $\pi \rightarrow \sigma^{*}$-quantum transitions. Moreover, since the integral of the overlap between $\pi$ and $\sigma^{*}$ $\mathrm{MO}$ is close to zero, these quantum transitions significantly weakened. The quantum transitions which are forbidden by symmetry are marked with the dashed line.

We present the structure of four MO of those listed in Table 3 (see Scheme 2).
2.2. The Energy Structure and Conformation of Molecule. Consideration of symmetry of MO (Table 3) shows that $\sigma$ MO may relate to representations $A_{1}$ and $B_{1}$; on the other hand, $\pi$-MO may relate to representations $A_{2}$ and $B_{2}$. Quantum transitions between $\sigma-\mathrm{MO}$ and $\pi$-MO can be permitted by symmetry but forbidden by space (the overlap integral between $\sigma$-MO and $\pi$-MO is close to zero, since the MO are oriented in mutually perpendicular directions).

As $\sigma$-MO and $\pi$-MO may be responsible for the absorption and emission of the molecule, only $\sigma$-MO is responsible for the formation of dissociative potential surfaces and photodissociation processes. On the other hand, only $\pi$-MO processes are responsible for cis-trans-isomerization.

In this paper we consider the isomerization processes and their impact on radiative processes in the excited molecules.

In the case of trimethine dyes, three isomers can be isolated: trans (I), cis (II) and cis-cis (III) (see Scheme 3).

The trans-isomer is a flat, highly symmetric molecule, while the other isomers are characterized by low symmetry. Furthermore, cis-isomer has a small angle $\left(\sim 8^{\circ}\right)$ between the planes of the two rings and angles in the methine bridge increased (from left to right: about 128.653 and about 128.754, and the angle of trans is about 123.097). This provides a reduction in repulsion energy between hydrogen atoms, which would otherwise overlap.

The angles in the thrimethine bridge of the cis-cis-isomer from left to right: about $129.345^{\circ}, 133.993^{\circ}$, and $129.552^{\circ}$. Moreover, trimethine bridges are twisted, and the rings are almost not turned (see Scheme 4).

The binding energy of the electron system in the molecule of I is $-2.170 .9248 \mathrm{kcal} / \mathrm{mol}=-94.14 \mathrm{eV}$, that of molecule II is $-2.170 .7688 \mathrm{kcal} / \mathrm{mol}=-94.1336 \mathrm{eV}$, and that of molecule III is $-2.165 .0604 \mathrm{kcal} / \mathrm{mol}=-93.8861 \mathrm{eV}$. If we expand cisisomer II bridge at about 90, we define the energy of the transition state to be equal to $-2.148 .3517 \mathrm{kcal} / \mathrm{mol}$. So, we can assume that the first two isomers in thermodynamic equilibrium will be equally represented in the dye solution, whereas the third isomer, the binding energy of which is at least $5.3084 \mathrm{kcal} / \mathrm{mol}=0.23 \mathrm{eV}$, shall be present in small amounts $\left(1 \cdot 10^{-4}\right)$. However, this fact does not exclude the involvement of this isomer in the process of photoisomerization.

In the process of conversion of the isomer I into isomer II the magnitude of the molecule dipole moment varies, as shown in Figure 1.

Since we are considering cation (I), then charge distribution in it, which determines the magnitude of the dipole moment, will determine the structure of the solvation shell in the dye solution, and this in turn may affect the structure of the isomer and its interaction with the solvent, further stabilizing the isomer.

In the case where isomer III dipole moment is $1.4135 \mathrm{D}$, during the transition from isomer I to isomer II, dependence of the dipole magnitude moment of the rotation angle around $\mathrm{C}-\mathrm{C}$ bond is almost symmetric, reaching the maximum value at 90-degree orientation of fragments of the molecule. Enlarged magnitude of dipole moment in both cases of isomerization at 90-degree orientation of the molecule fragments is indicative of the possibility of increasing the energy of the transition state interactions with solvent molecules, 
TABLE 2: Symmetric vibrations of the molecule (I).

\begin{tabular}{|c|c|c|c|}
\hline Number & $\begin{array}{l}\text { Frequency, } \\
\mathrm{cm}^{-1}\end{array}$ & Localization of vibrations & Intensity \\
\hline 1 & 70 & Two polynomial carried pendulum oscillations, changing the angle of the $\mathrm{C}$-C-C-center & 0.049 \\
\hline 2 & 300 & Folding: fragments move forward; methine bridge changes the angles & 0.078 \\
\hline 3 & 405 & Methine bridge and ring in antiphase & 0.011 \\
\hline 4 & 829 & Synchronous changing of angles $\mathrm{O}-\mathrm{C}-\mathrm{N}$ and central C-C-C & 0.29 \\
\hline 5 & 927 & Vibrations angle $\mathrm{O}-\mathrm{C}-\mathrm{C}$ in rings & 2.1 \\
\hline 6 & 1067 & Vibrations of methine angles bond and length $\mathrm{O}-\mathrm{C}-\mathrm{N}$ & 0.084 \\
\hline 7 & 1147 & Pendulum oscillations of $\mathrm{C}$ - $\mathrm{H}$ groups in the rings & 24 \\
\hline 8 & 1196 & Vibrations of the molecule along $y$-axis. & 0.23 \\
\hline 9 & 1280 & Vibrations lengths S-O-S & 2.5 \\
\hline 10 & 1320 & Pendulum oscillations of $\mathrm{N}-\mathrm{H}$ are accompanied by vibrations of the bonds and the methine bridge & 2.0 \\
\hline 11 & 1325 & Symmetric pendulum vibrations of $\mathrm{C}-\mathrm{H}$ bonds bridge & 2.4 \\
\hline 12 & 1418 & Vibrations rings along $x$; central heating $\mathrm{C}$ atoms along $y$. & 0.63 \\
\hline 13 & 1512 & Horizontal vibrations $\mathrm{C}-\mathrm{N}$ & 0.096 \\
\hline 14 & 1590 & The length of the C-N and C-C-C & 16 \\
\hline 15 & 1686 & $\mathrm{C}-\mathrm{C}$ rings & 3.8 \\
\hline 16 & 1864 & Bonds rings with the methine bridge & 44 \\
\hline 17 & 3129 & All three groups $\mathrm{C}-\mathrm{H}$ bridge in antiphase with the central $\mathrm{C}-\mathrm{H}$ & 17 \\
\hline 18 & 3162 & All three $\mathrm{C}-\mathrm{H}$ groups in the bridge phase & 70 \\
\hline 19 & 3207 & Two upper C-H bonds in the ring & 150 \\
\hline 20 & 3254 & Two lower $\mathrm{C}-\mathrm{H}$ bonds in the ring & 53 \\
\hline 21 & 3497 & Two N-H- bonds & 180 \\
\hline
\end{tabular}

TABLE 3: Symmetry MO.

\begin{tabular}{lc}
\hline Number of MO & Symmetry and the type of MO \\
\hline 29 & $\mathbf{A}_{\sigma-1} \mathrm{MO}$ \\
30 & $B_{2} \pi-\mathrm{MO}$ \\
31 & $A_{2} \pi-\mathrm{MO}$ \\
32 & $B_{2} \pi-\mathrm{MO}$ \\
33 & $A_{2} \pi-\mathrm{MO}$ \\
34 & $B_{2} \pi-\mathrm{MO}$ \\
35 & $A_{2} \pi-\mathrm{MO}$ \\
36 & $B_{2} \pi-\mathrm{MO}$ \\
37 & $A_{2} \pi-\mathrm{MO}$ \\
38 & $\mathbf{A}_{\sigma-1} \mathrm{MO}$ \\
39 & $\mathbf{B}_{\mathbf{1}} \sigma-\mathrm{MO}$ \\
40 & $\mathbf{A}_{\sigma-1} \mathrm{MO}$ \\
41 & $\mathbf{B}_{\mathbf{1}} \sigma-\mathrm{MO}$ \\
42 & $B_{2} \pi-\mathrm{MO}$ \\
43 & $\mathbf{A}_{\sigma-1} \mathrm{MO}$ \\
\hline
\end{tabular}

thus reducing the height of the potential barrier to the dark transition between the isomers.

Investigation of the energy structure of the isomers in excited states (Figures 2 and 3) showed that in the ground state there is a barrier between the two isomers, with height of $20.5 \mathrm{kcal} / \mathrm{mol}=0.89 \mathrm{eV}$. This value may be somewhat reduced due to the interaction with the solvent. However, this value
TABLE 4: The polarization of the quantum transitions between MO.

\begin{tabular}{lcccccccccc}
\hline MO & 34 & 35 & 36 & 37 & 38 & 39 & 40 & 41 & 42 & 43 \\
\hline 29 & $Z$ & - & $z$ & - & $\mathbf{y}$ & $\mathbf{x}$ & $\mathbf{y}$ & $\mathbf{x}$ & $z$ & $\mathbf{y}$ \\
30 & $\mathbf{Y}$ & $\mathbf{x}$ & $\mathbf{y}$ & $\mathbf{x}$ & $z$ & - & $z$ & - & $\mathbf{y}$ & $z$ \\
31 & $\mathbf{x}$ & $\mathbf{y}$ & $\mathbf{x}$ & $\mathbf{y}$ & - & $z$ & - & $z$ & $\mathbf{x}$ & - \\
32 & $\mathbf{y}$ & $\mathbf{x}$ & $\mathbf{y}$ & $\mathbf{x}$ & $z$ & - & $z$ & - & $\mathbf{y}$ & $z$ \\
33 & $\mathbf{x}$ & $\mathbf{y}$ & $\mathbf{x}$ & $\mathbf{y}$ & - & $z$ & - & $z$ & $\mathbf{x}$ & -
\end{tabular}

indicates a very low rate of establishment of thermodynamic equilibrium between the isomers.

Excitation of molecule (I) in $S_{1}$-state will lead to a decrease in the barrier height to $2.39 \mathrm{kcal} / \mathrm{mol}=0.104 \mathrm{eV}$. With such magnitude of barrier, (Boltzmann factor is 0.016 ) photoisomerization will compete with radiating process, whereby the long-wavelength fluorescence quantum yield of polymethine dyes does not exceed $0.55[15,16]$.

Figure 2 shows the data for the quantum transitions into the singlet-excited state up to the highest $\pi$-MO number 42 , because it has a disintegrating character for $\mathrm{C}-\mathrm{C}$ bonds of trimethine fragment. However, this $\mathrm{MO}$ did not contribute to cis-trans-isomerization of molecule (I), as its disintegrating character stayed for the second isomer.

As for the triplet states, excited $T_{5}$ state corresponding to the excitation of an electron from $\pi$-MO 33 on $\sigma$-MO 38 can cause isomerization of the molecule. Its intersection with state $T_{4}\left(\pi \rightarrow \pi^{*} \mathrm{MO}\right.$ transition between number 




MO number 33

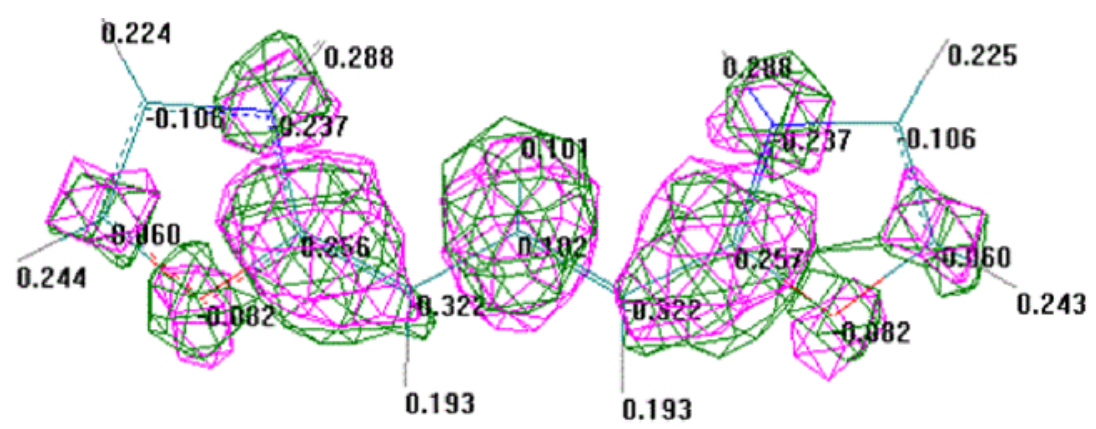

MO number 34

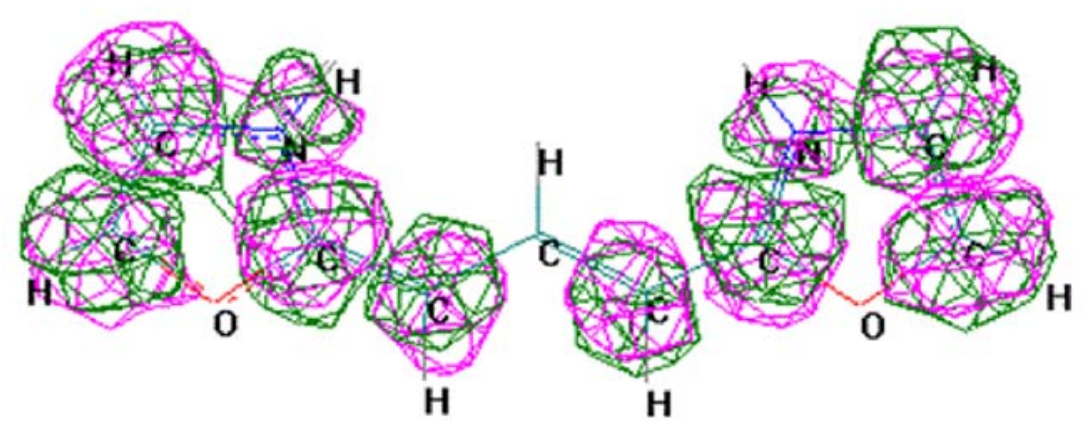

MO number 37

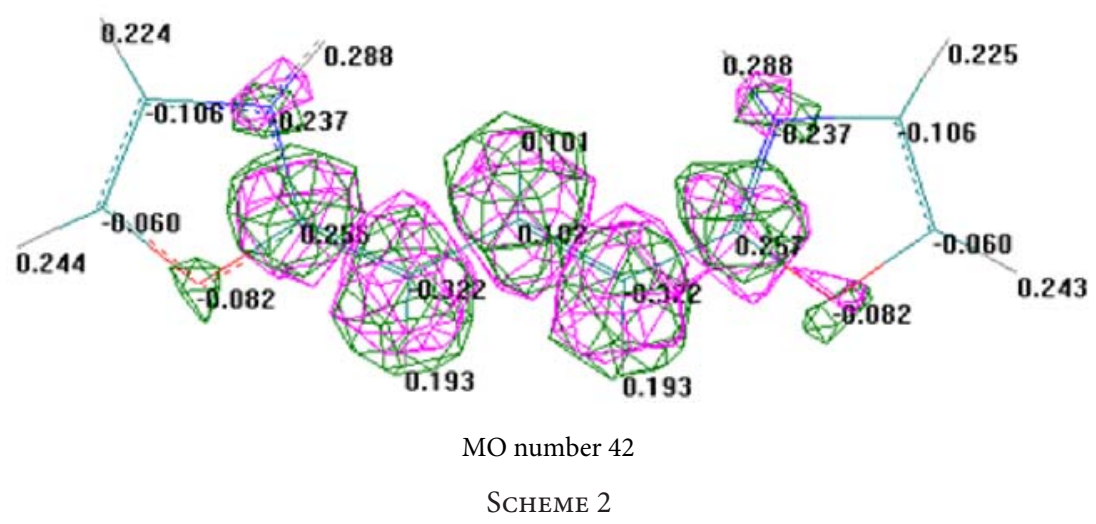


<smiles></smiles>

(I) trans-Isomer<smiles>C(=CC1CCCO1)c1ccco1</smiles>

(II) cis-Isomer<smiles>C1=COc2[nH]ccc2C=C1</smiles>

(III) cis-cis-Isomer

SCHEMe 3

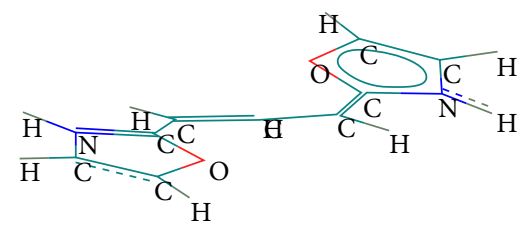

(Molecule III view on top)

SCHEMe 4

$33 \rightarrow$ numbers 35 and 37), which led to the splitting of the energy levels, should not significantly affect the ability to flow isomerization from this state. Interaction between these triplet states has become possible because the rotation of one relative to the other moiety of the molecule greatly reduced the symmetry of the molecule and mixed $\pi$ - and $\sigma$-MO.

Reference [9] noted that, under the influence of light, a photoisomer is created and long-wavelength band is shifted to lower energies with a short lifetime $\left(10^{-4} \div 10^{-3} \mathrm{sec}\right)$; at that, photoisomer is formed with low quantum yield. However, the main channel of decrease of the fluorescence quantum yield is considered nonradiative dissipation of excitation energy.

The results of this work testify that the first two isomers are stable and have $S_{0} \rightarrow S_{1}$-absorption bond in the same spectral region, and the third one may be unstable, and its corresponding absorption band is shifted to longer wavelengths.

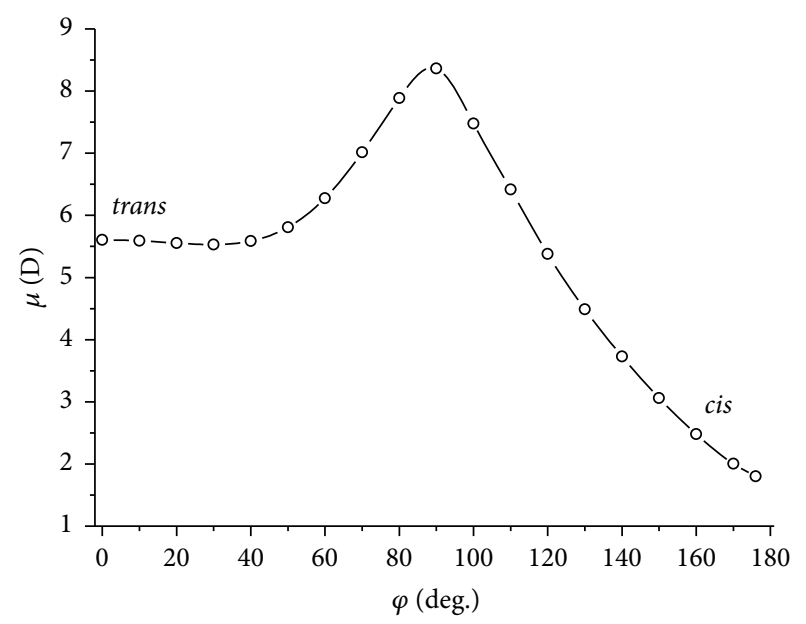

FIGURE 1: The dependence of the magnitude of dipole moment of the molecule (I) from the rotation angle of the molecule fragments around $\mathrm{C}-\mathrm{C}$ in the methine group.

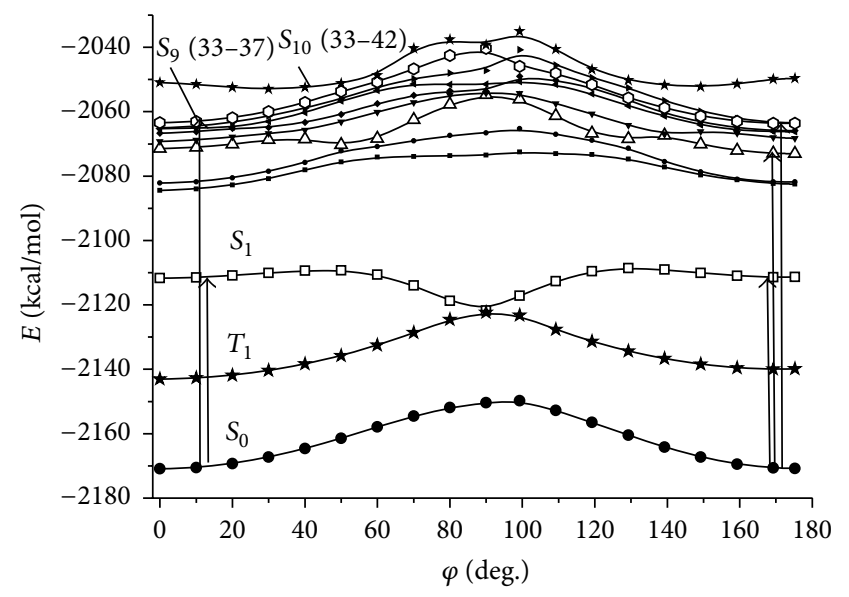

FIGURE 2: The angular dependence of the energy of the ground and excited singlet states of the molecule (I) isomer in the transition from I to II isomer.

Photoisomerization processes between the first two isomers will enhance the probability of nonradiative transitions, since the 90-degree orientation of fragments of the molecule and the energy gap between $S_{0}$ and $S_{1}$ are significantly reduced. Nonradiative processes should behave analogously at the transition from the second isomer to the third one. In all the these processes, the probability of the third isomer will be insignificant, since the excitation of the first isomer as a result will lead to the creation of equal probability of the first and second isomers, and the excitation of the second isomer can lead to the creation of all three isomers. As a consequence, a third isomer is created with a small quantum yield.

In the case of the plane geometry of molecule (I) in the absorption spectrum bands corresponding to the transition between quantum $\mathrm{MO}$ numbers 33 and 34 (long-wavelength absorption band) as well as between MO numbers 33 and 37 should appear (Figure 2). Other quantum transitions that lie between these transitions are characterized by a 
TABLE 5: Singlet-singlet quantum transitions in isomers I and II.

\begin{tabular}{|c|c|c|c|}
\hline \multicolumn{2}{|l|}{ trans-Isomer } & \multicolumn{2}{|l|}{ cis-Isomer } \\
\hline Type of transition & $l, \mathrm{~nm},(f)$ & Type of transition & $l, \mathrm{~nm},(f)$ \\
\hline$S_{0} \rightarrow S_{1} \pi \rightarrow \pi^{*}(33 \rightarrow 34)$ & $482.7(1.0418)$ & $\pi \rightarrow \pi^{*}(33 \rightarrow 34)$ & $481.0(0.8252)$ \\
\hline$S_{0} \rightarrow S_{2} \pi \rightarrow \sigma^{*}(33 \rightarrow 38)$ & $330.7(0.0000)$ & $\pi \rightarrow \sigma^{*}(33 \rightarrow 3833 \rightarrow 3932 \rightarrow 39)$ & $324.0(0.0011)$ \\
\hline$S_{0} \rightarrow S_{3} \pi \rightarrow \sigma^{*}(33 \rightarrow 3932 \rightarrow 38)$ & $322.1(0.0064)$ & $\pi \rightarrow \sigma^{*}(33 \rightarrow 3833 \rightarrow 3932 \rightarrow 38)$ & $321.5(0.0059)$ \\
\hline $\mathrm{S}_{0} \rightarrow \mathrm{S}_{4} \pi \rightarrow \pi^{*}(33 \rightarrow 3532 \rightarrow 34)$ & $287.4(0.0141)$ & $\pi \rightarrow \pi^{*}(33 \rightarrow 35)$ & $292.6(0.1068)$ \\
\hline$S_{0} \rightarrow S_{5} \pi \rightarrow \sigma^{*}(33 \rightarrow 4032 \rightarrow 41)$ & $281.2(0.0000)$ & $\pi \rightarrow \sigma^{*}(33 \rightarrow 4033 \rightarrow 41)$ & $279.0(0.0031)$ \\
\hline$S_{0} \rightarrow S_{6} 3 \pi \rightarrow \sigma^{*}(3 \rightarrow 4132 \rightarrow 40)$ & $274.3(0.0000)$ & $\pi \rightarrow \pi^{*}(32 \rightarrow 34)$ & $273.5(0.0228)$ \\
\hline$S_{0} \rightarrow S_{7} \pi \rightarrow \pi^{*}(33 \rightarrow 3532 \rightarrow 34)$ & $270.5(0.0121)$ & $\pi \rightarrow \sigma^{*}(33 \rightarrow 41)$ & $272.7(0.0160)$ \\
\hline$S_{0} \rightarrow S_{8} \pi \rightarrow \pi^{*}(33 \rightarrow 36)$ & $269.9(0.0285)$ & $\pi \rightarrow \pi^{*}(33 \rightarrow 3633 \rightarrow 37)$ & $266.7(0.1628)$ \\
\hline$S_{0} \rightarrow S_{9} \pi \rightarrow \pi^{*}(33 \rightarrow 37)$ & $266.0(0.3301)$ & $\pi \rightarrow \pi^{*}(33 \rightarrow 3732 \rightarrow 36)$ & $266.3(0.0772)$ \\
\hline$S_{0} \rightarrow S_{10} \pi \rightarrow \pi^{*}(33 \rightarrow 42)$ & $238.3(0.0000)$ & $\pi \rightarrow \pi^{*}(31 \rightarrow 34)$ & $236.0(0.0139)$ \\
\hline$S_{0} \rightarrow S_{11} \pi \rightarrow \pi^{*}(31 \rightarrow 34)$ & $235.9(0.0123)$ & $\pi \rightarrow \pi^{*}(33 \rightarrow 42)$ & $232.0(0.0897)$ \\
\hline
\end{tabular}

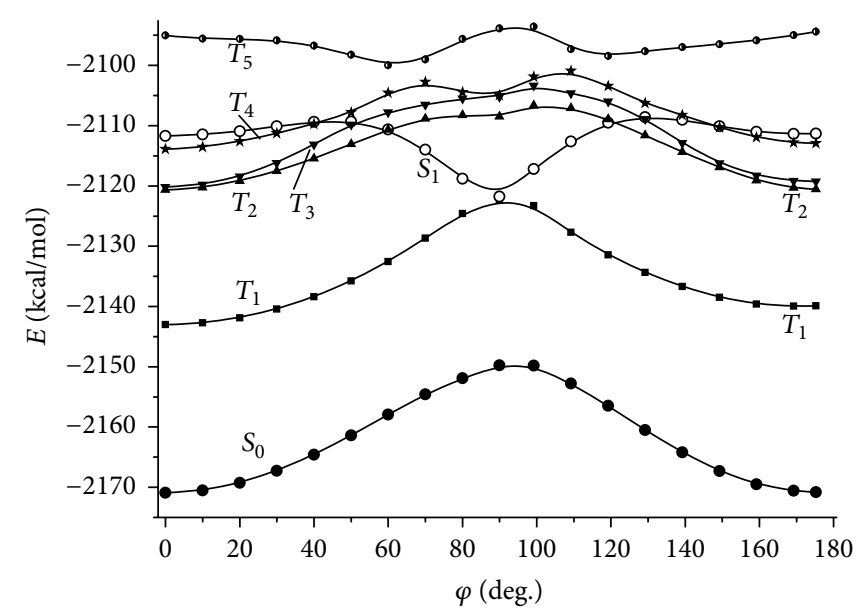

FIgURE 3: The angular dependence of the energy of the ground and triplet excited states of the molecule (I) isomer in the transition from I to II isomer.

small oscillator strength and therefore do not appear in the absorption spectra.

In isomer II, the long-wavelength absorption band is in the same spectral region as that in isomer I, but instead of a single absorption band, noted above, there are two bands (Table 5), since in this case the oscillator strength $\pi \rightarrow \pi^{*}$ transition is between MO numbers 33 and 35 which increased by almost an order of magnitude.

For us it is important to determine which quantum transition may be responsible for the short-wave fluorescence band.

Analysis of the nature of the second (apparent) absorption band indicates that the corresponding $\pi \rightarrow \pi^{*}(33 \rightarrow$ 37) quantum transition cannot provide the fluorescence of the highly excited states, as other $\pi \rightarrow \pi^{*}$ transitions are placed below.

For fluorescence the transition $S_{4} \rightarrow S_{0}$ might be responsible, but in this case the large Stokes shift (increased $2.800 \mathrm{~cm}^{-1}$ ) must be observed. Relative of this transition following the transition $S_{3} \rightarrow S_{0}$ (of $\pi \sigma^{*}$-state) is shifted to
$3750 \mathrm{~cm}^{-1}$, which, given the spatial band, will not contribute to rapid internal conversion. By analyzing the experimental results, it can be seen that high-energy band fluorescence is really characterized by a large Stokes shift $[17,18]$. Consequently, we conclude that this is responsible for $\pi \rightarrow \pi^{*}$ (33 $\rightarrow$ 35) transition.

We have considered above the totally symmetric vibrations of molecule (I), which will give the structure of longwavelength band in the absorption spectrum. The transition of the molecule to the other geometric structures (conformations) is accompanied by a significant lowering of the symmetry of the molecules. This will facilitate the possibility of manifestation of the electronic absorption spectrum of other vibrational frequencies of the molecule and, consequently, change the shape of the long-wavelength absorption band.

\section{Conclusions}

On the basis of the conducted researches of energetic structure and processes of photoisomerization of trimethine cyanine dye (I) we can conclude the following:

(1) Molecule (I) may reside in three different conformational states, one of which is highly symmetric (symmetry $C_{2 v}$ ) and the other two with low symmetry. In this case, two conformers (trans- and cis-conformers) have in the ground state almost the same energy of the electronic system and the same position longwavelength absorption band. The third conformer is characterized by lower $0.23 \mathrm{eV}$ binding energy of the electronic system, and the long-wavelength absorption band is shifted to lower energies.

(2) The group theoretical analysis of molecule (I) allowed systematizing the vibrational and electronic quantum transitions and identifying the bands in the absorption spectra.

(3) It is shown that the excitation of the molecule in $S_{1}$ state causes trans-cis-isomerization. The presence of the barrier height of $\sim 0.1 \mathrm{eV}$ allows the process fluorescence to compete with isomerization process, but isomerization causes a decrease in the fluorescence 
quantum yield of the dye. Since there are two stable isomers and one unstable isomer, photoisomerization processes with low quantum yield lead to the creation of unstable isomer. The probability of nonradiative processes on each channel increases, which reduces the fluorescence quantum yield of the dye in the isomerization process.

(4) Investigation of the absorption spectra of the isomers in the UV region of the spectrum showed that in the isomer of I a single absorption band manifested with a large value of the oscillator strength $(f=0.33)$, the excitation of which can fix the short-wavelength fluorescence with a significant Stokes shift. Calculation showed that close to this absorption band at lower energies there are other quantum transitions with small oscillator strength, that pertain both to a $\pi \rightarrow \pi^{*}$ and to $\pi \rightarrow \sigma^{*}$ quantum transitions. In the second isomer, one of such $\pi \rightarrow \pi^{*}$ transitions have sufficient appreciable oscillator strength $(f=0.1)$, whereas in the first one, there is a less order of magnitude. Relaxation of excitation in this state may result in a large Stokes shift of high energy fluorescence.

\section{Conflict of Interests}

The authors declare that there is no conflict of interests regarding the publication of this paper.

\section{References}

[1] J. M. Hales, J. Matichak, S. Barlow et al., "Design of polymethine dyes with large third-order optical nonlinearities and loss figures of merit," Science, vol. 327, no. 5972, pp. 1485-1488, 2010.

[2] A. Mishra, R. K. Behera, P. K. Behera, B. K. Mishra, and G. B. Behera, "Cyanines during the 1990s: a review," Chemical Reviews, vol. 100, no. 6, pp. 1973-2012, 2000.

[3] N. Peyghambarian, L. Dalton, J. Alex et al., "Technological advances brighten horizons for organic nonlinear optics," Laser Focus World, vol. 42, no. 8, pp. 85-92, 2006.

[4] T. D. Iordanov, J. L. Davis, A. M. E. Masunov, A. Levenson, O. V. Przhonska, and A. D. Kachkovski, "Symmetry breaking in cationic polymethine dyes, part 1: ground state potential energy surfaces and solvent effects on electronic spectra of streptocyanines," International Journal of Quantum Chemistry, vol. 109, no. 15, pp. 3592-3601, 2009.

[5] O. V. Przhonska, S. Webster, L. A. Padilha et al., Advanced Fluorescence Reporters in Chemistry and Biology I: Fundamentals and Molecular Design, Springer Series in Fluorescence, Springer, Berlin, Germany, 2010.

[6] I. G. Davidenko, Yu. L. Slominskiy, A. D. Kachkovskiy, and A. I. Tolmachev, "Polymethine dyes-the derivatives of 7,8-dihydrobenzo [cd]-furo [2,3-f] indole," Ukrainian Chemical Journal, vol. 74, no. 4, pp. 105-113, 2008 (Russian).

[7] A. V. Kulinich and A. A. Ischenko, "Merotsianinovyie krasiteli: sintez, stroenie, svoystva, primenenie," Uspehi himii, vol. 78, no. 2, pp. 151-175, 2009 (Russian).

[8] A. H. Tarnovskiy, T. K. Razumova, E. P. Schelkina, and T. V. Veselova, "Photophysical, photochemical, and lasing characteristics of symmetric and asymmetric di- and trikarbotsianin dyes," Optics and Spectroscopy, vol. 74, pp. 107-115, 1993 (Russian).

[9] M. J. S. Dewar, E. G. Zoebisch, E. F. Healy, and J. J. P. Stewart, "AM1: a new general purpose quantum mechanical molecular model," Journal of the American Chemical Society, vol. 107, no. 13, pp. 3902-3909, 1985.

[10] P. A. Kondratenko, S. Y. Lopatkin, Y. M. Lopatkin, and T. N. Sakun, "Fotoelektricheskie svoystva polimernyih sloyov s krasitelyami," Visnik Sums'kogo Derzhavnogo Universitetu, vol. 1, pp. 145-153, 2007 (Russian).

[11] P. O. Kondratenko, Yu. M. Lopatkin, and T. M. Sakun, "Quasiequilibrium processes in the high excited molecules of resazurin," Journal of Nano- and Electron Physics, vol. 4, no. 2, Article ID 02017, 7 pages, 2012 (Ukraine).

[12] P. O. Kondratenko, Yu. M. Lopatkin, and T. M. Sakun, "Relaxation processes in the high-excited molecules of resazurin," Physics and Chemistry of Solid State, vol. 8, no. 1, pp. 100-108, 2007 (Ukrainian).

[13] R. J. Cave, K. Burke, and E. W. Castner Jr., “Theoretical investigation of the ground and excited states of coumarin 151 and coumarin 120," Journal of Physical Chemistry A, vol. 106, no. 40, pp. 9294-9305, 2002.

[14] S. Kumar, S. K. Jain, and R. C. Rastogi, "An experimental and theoretical study of excited-state dipole moments of some flavones using an efficient solvatochromic method based on the solvent polarity parameter, $E_{T}^{N}$," Spectrochimica Acta Part A: Molecular and Biomolecular Spectroscopy, vol. 57, no. 2, pp. 291298, 2001.

[15] D. Peceli, H. Hu, D. A. Fishman et al., "Enhanced intersystem crossing rate in polymethine-like molecules: sulfur-containing squaraines versus oxygen-containing analogues," Journal of Physical Chemistry A, vol. 117, no. 11, pp. 2333-2346, 2013.

[16] V. I. Zemskiy, Y. U. L. Kolesnikov, and I. K. Meshkovskiy, Physics and Technology of Pulsed Dye Lasers, Petersburg State University of Information Technologies, Saint Petersburg, Russia, 2005 (Russian).

[17] S. Webster, L. A. Padilha, H. Hu et al., "Structure and linear spectroscopic properties of near IR polymethine dyes," Journal of Luminescence, vol. 128, no. 12, pp. 1927-1936, 2008.

[18] G. G. Dyadyusha, O. V. Przhonskaya, Y. A. Tikhonov, and M. T. Shpak, "The fluorescence intensity out the second excited state of molecular solutions of organic dyes," Journal of Experimental and Theoretical Physics, vol. 1, no. 14, pp. 330-333, 2008 (Russian). 

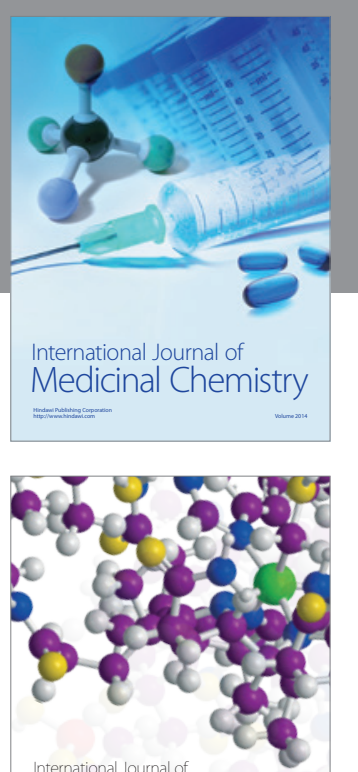

Carbohydrate Chemistry

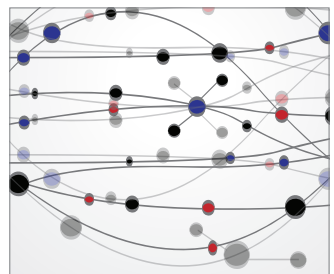

The Scientific World Journal
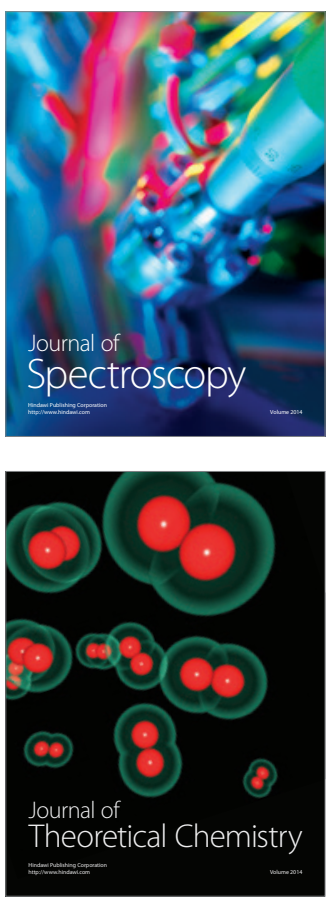
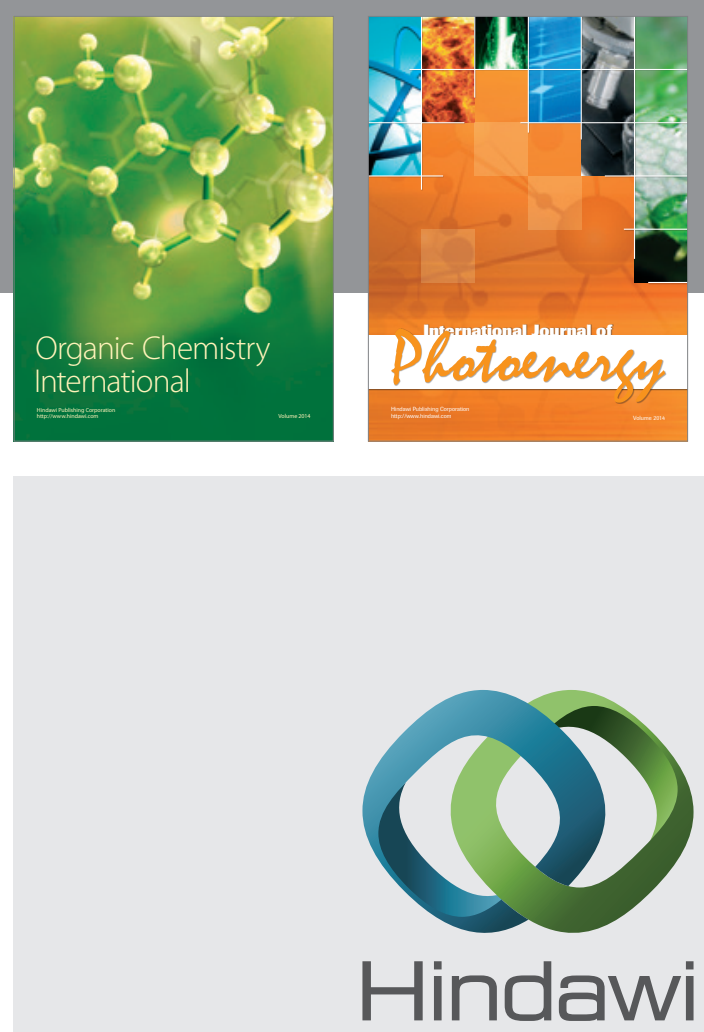

Submit your manuscripts at

http://www.hindawi.com

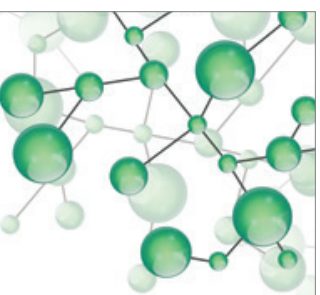

International Journal of

Inorganic Chemistry

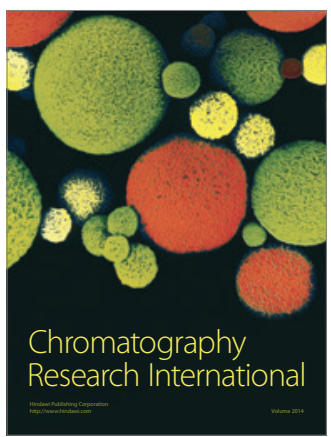

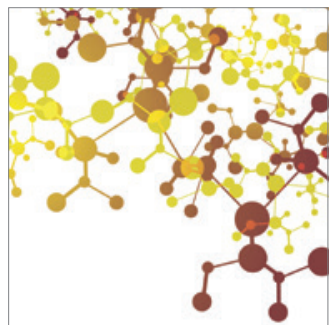

Applied Chemistry
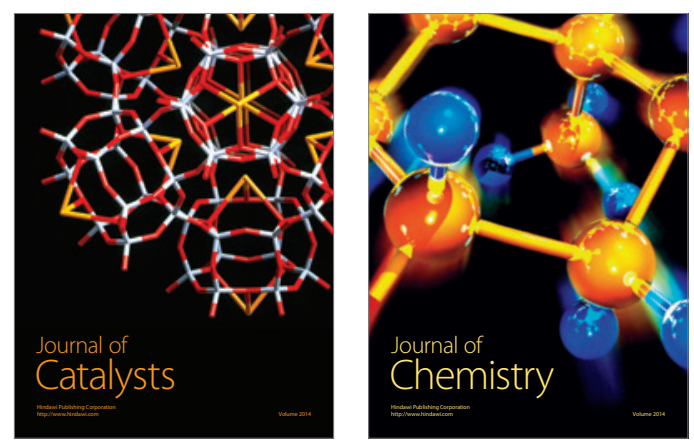
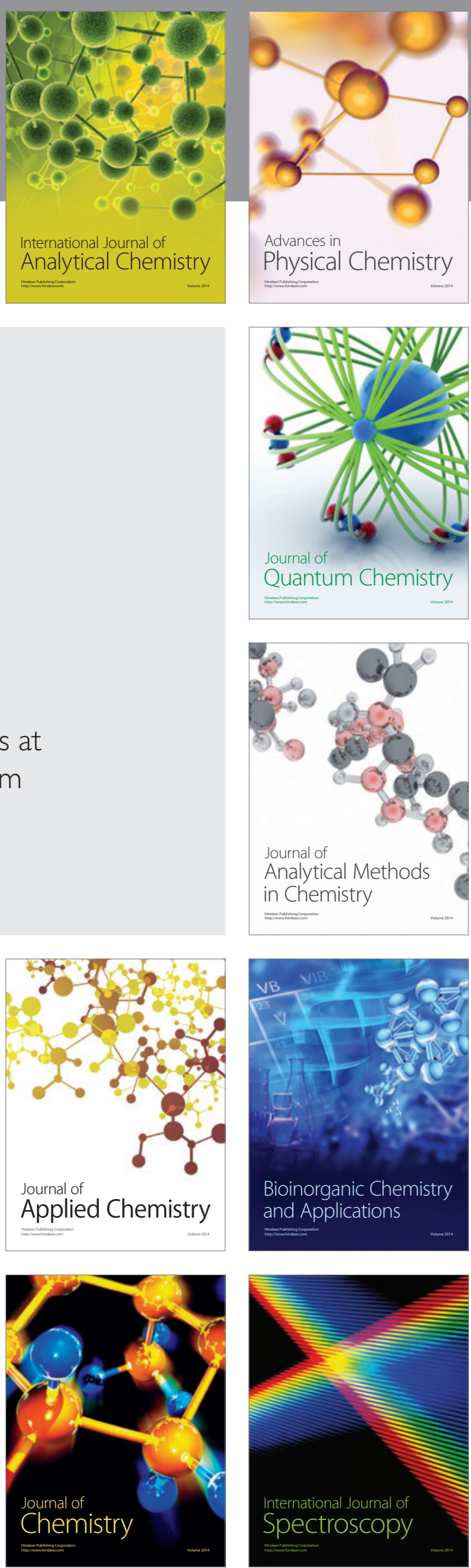